\title{
Integrating Aspects of Supply Chain Design into the Global Sourcing Process - Insights from the Automotive Industry
}

\author{
Gregor von Cieminski ${ }^{1}$ and Alessandro Rossi ${ }^{2}$ \\ ${ }^{1}$ ZF Friedrichshafen AG, Friedrichshafen, Germany \\ ${ }^{2}$ ETH Zurich, BWI Center for Industrial Management, Zurich, Switzerland \\ gregor.cieminski@zf.com, alrossiastudent.ethz.ch
}

\begin{abstract}
This paper reports on enhancements of the global sourcing process proposed with the purpose of increasing the weight of criteria related to supply chain design in sourcing decisions. In a conceptual study carried out for a German automotive supplier, the global sourcing process proposed by Alard et al. was found to be most suitable for industrial application. From the technical perspective of the supply chain management function, even this comprehensive approach does not address important decision criteria, such as likely structure of global supply chains or the capacity flexibility required of suppliers in global supply chains. The enhancements proposed include new process steps making use of network optimization and simulation techniques to eliminate these shortcomings.
\end{abstract}

Keywords: Global sourcing, supply chain design, network optimization, simulation.

\section{Introduction}

In the same way as many other industries, the automotive sector is going through a phase of global expansion. Industry surveys provide evidence that market growth in the industry is shifting from its traditional strongholds in the western industrialized world to the emerging markets, especially in the Asia-Pacific region. The CEOs of automotive companies expect China to remain the specific national market that offers the biggest growth potentials [1]. This country alone is predicted to provide around $30 \%$ of the global production of light passenger vehicles in 2020 [2].

Among other trends, these market developments are driving two changes in the global structure of the automotive industry. On the one hand, western OEMs (Original Equipment Manufacturers) and suppliers are forced to increase production in emerging markets in order to fulfil their growth objectives. On the other hand, automotive companies entering the market from the emerging nations have increased the cost pressure on their western competitors.

As a consequence, western automotive companies have increased the share of purchasing volume sourced from emerging markets. This is mainly for two reasons: 
1. Suppliers in emerging markets (or NSMs, short for "new souring markets") offer purchasing price advantages over the local or regional suppliers in Europe or the United States.

2. In their drive for global growth, western automotive companies aim to avoid the high transaction costs from their traditional production and supply base into the emerging markets. Therefore, new production facilities and a new supply base have to be built up in the emerging markets, i. e. on a global scale.

ZF Friedrichshafen AG (from here on referred to as "ZF"), a German automotive supplier, is also subject to the industry trends described above. In the specific market environment of the organisation, the importance of China as an NSM is growing. Given the growth of supply volumes sourced in China, the Supply Chain Management (SCM) function in the Asia-Pacific region is facing increasing difficulties in operating ZF's regional supply chains. For example, little attention may be paid to the impact of the location of a newly selected supplier on the structure of ZF's transportation network in China. The experience of the company confirms the findings of the analysis of Ruamsook et al.: SCM objectives are often assigned secondary importance in global sourcing decisions [3].

The SCM function of ZF in the Asia-Pacific region therefore initiated a study into possible improvements of the sourcing process [4]. This paper reports on the results of this study. First, it presents the case company and the methodology applied in, and the main findings of, the study. Second, it proposes enhancements of the global sourcing process from an SCM perspective in order to eliminate the shortcomings of the current sourcing practice. A set of general conclusions from the study completes the paper.

\section{Initial Analysis of Global Sourcing Practices}

This section first introduces the case company ZF Friedrichshafen AG. Second, the methodology applied in the analysis of the global sourcing practices of this company is presented and the main findings of the initial analysis are summarized.

\subsection{Case Company}

ZF Friedrichshafen AG is the third biggest German automotive supplier and a leading manufacturer of driveline and chassis technology. In 2013, ZF's total sales amounted to $€ 16.8$ billion and the company employed around 72,500 staff worldwide in its 122 production locations and 33 service centres [5]. ZF Group is organized in four divisions defined with an orientation towards customers as well as technologies provided by the company. Additionally, the group encompasses two cross-divisional business units. ZF produces a range of driveline and chassis components - such as transmissions, powertrain modules, axle systems, suspension systems - for the automotive, marine, railway, aviation and wind-power industries.

ZF has been operating international production facilities for a number of decades, opening its first plant outside of Europe in Brazil as early as 1958. In recent years, 
the company has accomplished accelerating growth on a global scale. The biggest growth rates are in the Chinese operations. Here, as in the other global regions, the company has established regional headquarters that support the regional plants by providing corporate services across the main business functions. The regional SCM office initiated the analysis of global sourcing processes illustrated in this paper.

\subsection{Methodology and Findings}

The methodology applied in the analysis of global sourcing processes at ZF and in the enhancement of these processes was the "Systems Engineering" approach developed by Haberfellner et al. [6]. The Systems Engineering approach is structured into four phases (corresponding to the system life cycle). This section summarises the different phases with reference to the analysis presented:

1. Trigger: The problem trigger of the analysis of global sourcing processes were the increasing difficulties the Chinese corporate SCM team in the Asia-Pacific region faced in operating ZF's regional supply chains. The purpose of the analysis was to identify possibilities to involve the SCM team earlier in the decision process in order to more systematically and effectively consider the concerns of the function in sourcing decisions.

2. System development: The system development phase is sub-divided into a preliminary study, a main study and further detailed studies. Each study uses a defined problem solving cycle. This is described with regards to the global sourcing process in more detail below.

3. System implementation: The subject of this phase is the technical system implementation, i. e. converting the system concept into a tangible object or executable process. This phase was not within the scope of the global sourcing study and is therefore not presented in this paper.

4. System use: The system use phase represents the continuous use and improvement of the system under real operating conditions. This phase is also not covered by this paper.

The remainder of the paper focuses on the process steps of the second phase of the Systems Engineering approach, system development. As stated above, the problem solving cycle defined by Haberfellner et al. [6] provided the procedural framework:

1. Situation analysis: The situation analysis mainly examined the global sourcing practices at $\mathrm{ZF}$ in the Asia-Pacific region. The analysis included the documentation of the sourcing processes and practices currently in use in the region, challenges and problems associated with regional sourcing decisions and a SWOT analysis of the processes. It was shown that ZF has implemented an advanced global sourcing process in terms of the tasks typically associated with the purchasing function (determining the supply demand, supply market research, supplier identification, supplier evaluation, supplier selection). The analysis also revealed that the process neglects SCM-oriented decision criteria. Thus, sourcing decisions are taken that have a detrimental effect on supply chain performance and supply chain costs. 
2. Objective formulation: For engineering the global sourcing process, the ZF SCM team Asia-Pacific defined the clear objective that aspects of supply chain design have to be integrated in the global sourcing process. The results of these analyses have to be considered as additional SCM-oriented criteria in the global sourcing decisions of the company.

3. Solution search: ZF's Asia-Pacific team engaged on a search for a blueprint on which to base improvements of the sourcing process of the company. The search considered reference processes for both, supply chain design and global sourcing, documented in industrial as well as scientific literature. A set of 10 relevant reference processes was thus identified and evaluated. Interestingly, a considerable number of process models from the field of purchasing were excluded because of their solely operational focus.

4. Evaluation: ZF based its evaluation of the 10 relevant reference processes on a set of defined criteria that covered both technical aspects (e.g. "Reference process includes procedure for rough supply chain design.") as well as application-oriented aspects (e.g. "Reference process includes clear documentation of process steps and their building blocks.") [4]. The criteria were weighted according to their importance and the corresponding scores determined for each of the reference processes under consideration.

5. Decision: As a result of the evaluation, the global sourcing process (GSP) developed Alard et al. [7, 8] was selected as the most suitable process reference for ZF. Figure 1 shows the sequence of fifteen steps Alard et al. have defined for GSP. Its advantages are explained with reference to the figure.

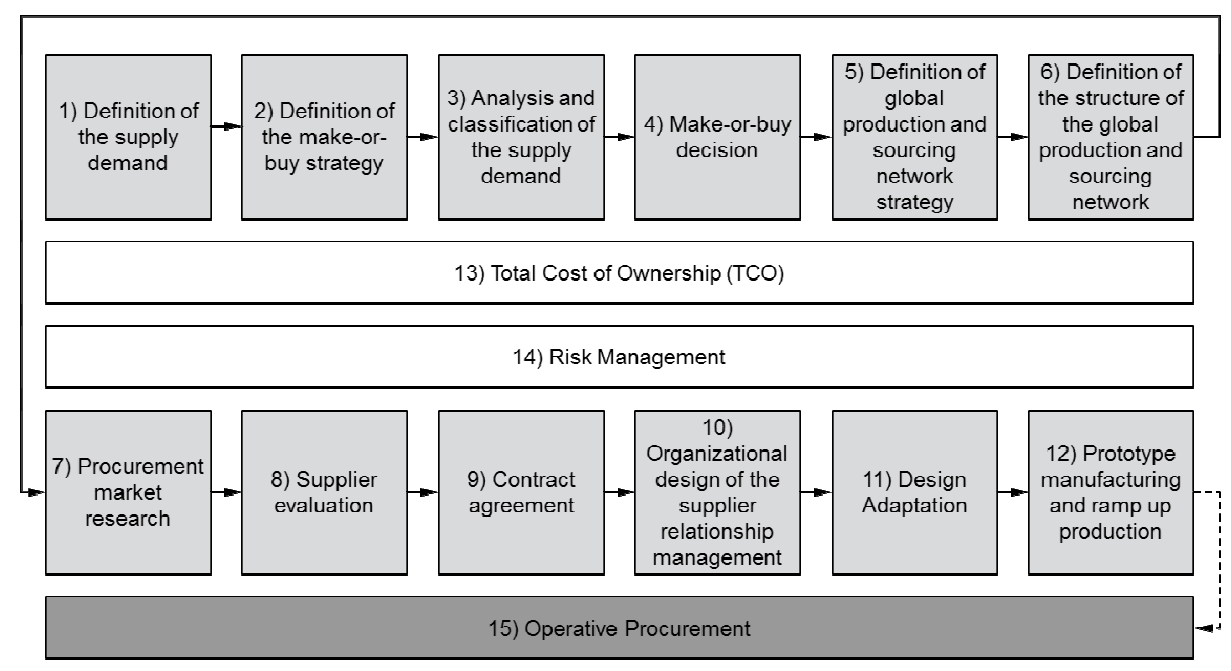

Fig. 1. Global sourcing process and its steps $[7,8]$

The main advantage of GSP is the inclusion of two explicit steps concerned with the design of the production and sourcing network (steps 5 and 6). This ensures that strategic SCM-oriented aspects are considered in the subsequent sourcing decisions. 
Also, if relevant, the GSP process steps integrate the total costs of ownership (TCO) as well as aspects of risk management in their analysis (steps 13 and 14, applicable along the chain of process steps). These elements guarantee a balanced consideration of relevant criteria for the sourcing decisions, including supply chain costs and risks.

\section{Enhancing the Global Sourcing Process}

Having selected GSP as the relevant process template, the purpose of the main study by ZF was two-fold. On the one hand, ZF matched the GSP process definitions with practices already applied by the company. On the other hand, the company analysed whether GSP matches all its requirements in detail. As this was not completely found to be the case, the process steps "static and dynamic analysis of the sourcing network" were newly defined. These are presented in section 3.1. Subsequent detailed studies were concerned with developing the process steps in more detail. As an example the process step "dynamic analysis of sourcing network" is described in section 3.2.

\subsection{Supply Chain Design Steps in the Overall Process}

Figure 2 depicts the GSP including the enhancements added by ZF.

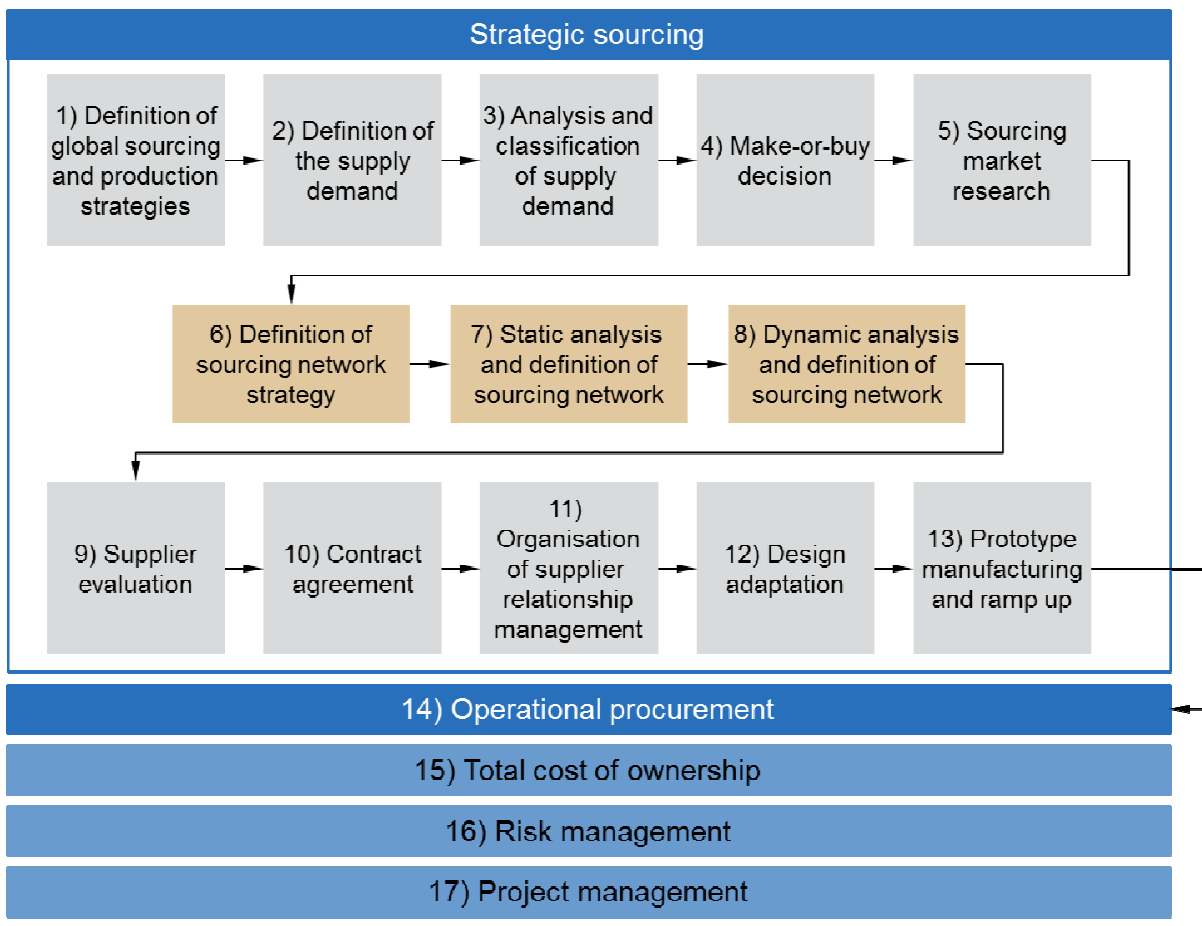

Fig. 2. Integration of supply chain design and global sourcing 
Evidently, ZF did not change the overall structure of GSP. Indeed, steps 9 to 16 remain the same as before. Step 17 "Project management" was included as an additional cross-process activity to reflect the nature of GSP decisions. Also, in steps 1 to 4 less emphasis was placed on the make-or-buy strategy and other production-related aspects.

The main enhancements are related to steps 5 to 8 (cf. figure 2). First, the process "Sourcing market research" is drawn forward (step 5). This is to reflect the fact that before any specific analyses can meaningfully be carried out, general information about the NSM under investigation ought to be collected. On the basis of this, a first "Sourcing network strategy" (step 6) may be defined that specifies common guidelines for supply chain design in the NSM.

Steps 7 and 8 represent the essential new steps integrated into GSP. Step 7 entails a "Static analysis of the sourcing network". By means of network optimisation models, the overall structure of the NSM supply chain is analysed. Two types of investigation are conceivable: First, a generic study of the NSM based on probable scenarios of the supply chain structures. This serves the purpose of a general examination of the characteristics and challenges for supply chain designs in the NSM. Overall guidelines for designing the structure of the supply chain network should become apparent. Second, specific studies of the supply chain structures related to a concrete sourcing case. The outcomes of this serve as critical inputs for the TCO analysis with regards to the potential suppliers.

Step 8 provides for a "Dynamic analysis of the sourcing network". On the basis of generic simulation models, the same kind of general as well as specific investigations as for step 7 can be carried out. The objective of the dynamic analysis is to predict the effects of unforeseen or unplanned events on the NSM supply chains. The definition of adequate responses to customer demand variations is of particular interest, namely the requirements for capacity flexibilities and safety stocks. By use of the dynamic analyses, the supply chain costs can be determined even accurately and actions to mitigate supply chain risks can be evaluated in detail.

\subsection{Use of Simulation for Dynamic Supply Chain Analysis of Sourcing Market}

Detailed studies for the newly proposed process steps represented the last stage of the system development phase for the global sourcing process. These were carried out for the static analysis and the dynamic analysis of the sourcing network. The ZF SCM reference model [9] provided a template for the process design. Figure 3 shows the procedure defined for the dynamic analysis of the sourcing network as an example.

Design of process step. The overall process step "Dynamic analysis of sourcing network" is split into 7 sub-process steps. The sequence of these follows that of general simulation studies. The simulation models are built in order to be able to study the NSM supply chains under different operating scenarios. ZF intends to use a supply chain simulation software tool available to the company as a means for the dynamic analysis. As can be deduced from the inputs defined for the sub-process steps (cf. figure 3), the simulation models will be implemented as a comparatively 
high-level representation of the real supply chains. This allows the simulation studies to focus on a limited set of critical dynamic characteristics.

Designated use of process step. There are two critical advantages of analysing feasible supply chains in new sourcing markets by means of dynamic events-based simulation. On the one hand, the critical supply chain characteristics - e.g. customer demand, capacity flexibility - may be considered from the perspective of medium- to long-term capacity planning. The Asia-Pacific SCM team can thus clearly state the basic SCM-oriented requirements and make sure that potential suppliers meet these. On the other hand, similar requirements can be formulated for worst-case scenarios, which also have to be fulfilled across the sourcing network. The impact of a basic set of supply chain risks - e.g. supplier failure, failure of the transport infrastructure - can be accounted for. By means of this, ZF is able to support efforts for supply chain risk management in the sourcing network.

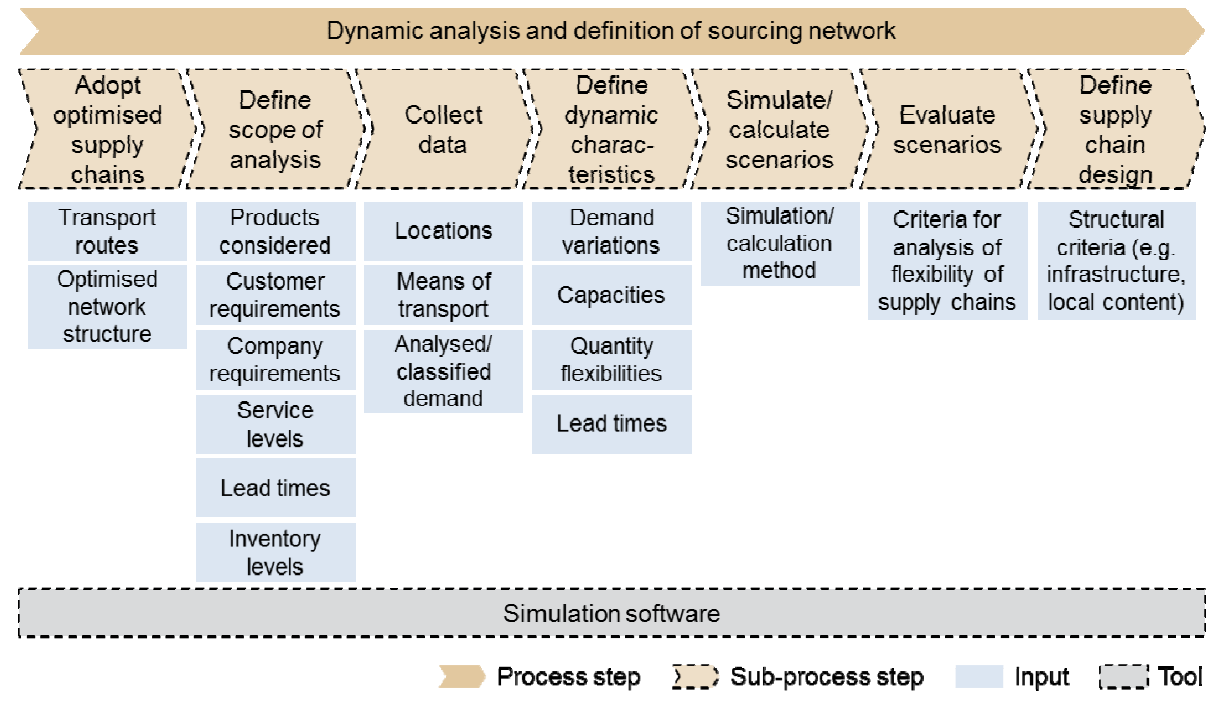

Fig. 3. Procedure for dynamic analysis of supply chains

Further development of process step. By putting the dynamic analysis of the sourcing network - as well as the other new process steps defined - into practice, ZF expects to obtain valuable information about the applicability of the design of the process steps. Most crucially, it should become apparent which characteristics of the NSM supply chains or sourcing networks need to be investigated in detail due to their criticality. Repeated applications of the enhanced GSP will thus lead to a continuously refined process design. 


\section{Conclusions}

An analysis of the global sourcing processes at ZF Friedrichshafen AG illustrated that the sourcing decision process of the company does not adequately consider SCMoriented aspects. The evaluation of relevant process models available in literature revealed these also to be lacking in this respect. For this reason, ZF enhanced the global sourcing process developed by Alard et al. by two specific steps: a static sourcing network analysis employing network optimisation approaches and a dynamic sourcing analysis on the basis of simulation experiments.

As a next step, ZF is planning a pilot application of the enhanced approach for global sourcing led by the corporate SCM team of the Asia-Pacific region. Clear recommendations on the practical design of the supply chains in China as an NSM are sought. At the same time, the practical applicability of the process enhancements presented in this paper will be evaluated. Specifically, the level of detail that is required of the investigation to generate meaningful results should be determined. In the future, cross-industry process benchmarks as well as rigorous action-based research (ensuring a formal scientific input) may be used to further improve the global sourcing practices.

\section{References}

1. PricewaterhouseCoopers: Fit for the Future: 17th Annual Global CEO Survey. Key findings in the automotive industry, London (2014)

2. IHS Global Insight: IHS Automotive Light Vehicle Production Forecast, Lexington, MA (2014)

3. Ruamsook, K., Russell, D.M., Thomchick, E.A.: Sourcing from low-cost countries: Identifying sourcing issues and prioritizing impacts on logistics performance. International Journal of Logistics Management 20(1), 79-96 (2009)

4. Rossi, A.: Impact of Future Sourcing Decisions on the Supply Chain Concepts for the Asian Region: Integrating Supply Chain Design Aspects in the Sourcing Process of ZF Friedrichshafen AG. Master thesis, BWI Center for Industrial Management, ETH Zurich (2014)

5. ZF Friedrichshafen AG: Annual Report 2013. Friedrichshafen (2014)

6. Haberfellner, R., de Weck, O.L., Fricke, E., Vössner, S.: Systems Engineering: Methodik und Praxis, 12th edn., OrellFüssli, Zürich (2012) (English edition forthcoming)

7. Alard, R., Bremen, P., Oehmen, J., Schneider, C.: Total Cost of Ownership Considerations in Global Sourcing Processes. In: Vallespir, B., Alix, T. (eds.) Advances in Production Management Systems. IFIP AICT, vol. 338, pp. 491-498. Springer, Heidelberg (2010)

8. Alard, R., Oehmen, J., Bremen, P.: Reference Process for Global Sourcing. In: Proceedings of 13th IEEE International Conference on Industrial Engineering and Engineering Management, pp. 367-371. IEEE, Singapore (2007)

9. von Cieminski, G.: Adapting and applying the Supply Chain Operations Reference model a case study from the automotive industry. In: Frick, J. (ed.) Advances in Production Management Systems (2011) 